[0212-7199 (2005) 22: 4; pp 188-190] ANALES DE MEDICINA INTERNA Copyright (C) 2005 ARAN EDICIONES, S.L

AN. MED. INTERNA (Madrid) Vol. $22, \mathrm{~N}^{\circ} 4$, pp. $188-190,2005$

\title{
El síndrome postpericardiotomía tras la implantación de marcapasos: presentación de un caso y revisión
}

\author{
J. A. NIETO RODRÍGUEZ, D. VAÑÓ SANCHIS, F. ALONSO PARDO, V. DE PAZ \\ VAREA
}

Departamento de Medicina Interna, Cardiología y Unidad de Cuidados Intensivos. Hospital Virgen de la Luz. Cuenca

THE POSTPERICARDIOTOMY SYNDROME FOLLOWING ENDOVENOUS PACEMAKER INSERTION: CASE REPORT AND REVIEW

\section{RESUMEN}

Presentamos un caso de síndrome postpericardiotomía tras la implantación de un marcapasos endovenoso (SPIV) en una mujer de 78 años y revisamos la experiencia publicada. Desde 1975 se han publicado en la literatura médica diez casos más de SPIV. La escasez de casos publicados y la alta tasa de complicaciones contrastan con la elevada prevalencia (4.6\%) y el curso benigno de las pericarditis agudas tras la inserción de marcapasos endovenosos en una serie de 126 pacientes consecutivos.

Esta clase de SPIV está probablemente infradiagnosticada y los casos publicados probablemente representan solo las formas clínicas más graves. Un alto índice de sospecha debería mejorar su diagnóstico y tratamiento.

PALABRAS CLAVE: Postcardiotomía. Postpericardiotomía. Marcapasos endovenoso. Pericarditis. Derrame pericárdico.

\begin{abstract}
We report a postpericardiotomy syndrome (PS) following the implantation of endovenous pacemaker in a 78-year-old woman. Ten more single cases were reported in the medical literature since 1975. The infrequent single reports and the high complication rate are in contrast with the 4,6\% occurrence and the benign course of acute pericarditis following the insertion of endovenous pacemakers in 126 consecutive patients. This sort of PS is probably underdiagnosed and the cases reported represent the more severe clinical presentations. A higher suspicion index would improve the diagnosis and management.
\end{abstract}

KEY WORDS: Postcardiotomy. Postpericardiotomy. Endovenous pacemaker. Pericarditis. Pericardial effusion.

Nieto Rodríguez JA, Vañó Sanchis D, Alonso Pardo F, de Paz, Varea V. El síndrome postpericardiotomía tras la implantación de marcapasos: presentación de un caso y revisión. An Med Interna (Madrid) 2005; 22: 188-190.

\section{INTRODUCCIÓN}

El síndrome postpericardiotomia (SP) se caracteriza por la combinación de fiebre, disnea y dolor pleurítico tras una lesión del pericardio (1). El SP se ha descrito tras cirugía cardiaca, infarto de miocardio (2), traumatismos torácicos cerrados, punción percutánea del ventrículo izquierdo, perforación coronaria durante la angioplastia con balón e implantación de marcapasos epicárdicos (3). También se ha descrito tras la colocación de marcapasos endovenosos (SPIV) aunque solo muy raramente, pues desde su primera descripción en 1975, sólo se han publicado diez casos (4-13) y una pequeña serie (14).

\section{CASO APORTADO}

Mujer de 78 años que fue remitida al hospital cuando se le detectó un bloqueo auriculoventricular de tercer grado durante un examen rutinario. La paciente quedó ingresada y se implantó un marcapasos temporal endovenoso 7F (electrodos de platino) en el ventrículo derecho a través de la vena yugular interna derecha. Fue necesario recolocarlo en cinco ocasiones durante las primeras 24 horas debido a un insuficiente sensado auricular. El tercer día de ingreso se colocó un marcapasos permanente VDD a través de la vía subclavia izquierda sin incidencias. Al día siguiente la paciente tuvo febrícula y fue tratada ambulatoriamente con cloxacilina. Los hemocultivos fueron negativos. Diez días mas tarde, ingresó de nuevo en el hospital debido a febrícula intermitente, disnea, tos no productiva y dolor pleurítico en hemitórax derecho. La herida quirúrgica estaba limpia e indolora. La radiografía de tórax reveló cardiomegalia y un derrame pleural derecho. El ECG mostraba un bloqueo auriculoventricular de tercer grado con ritmo de escape de marcapasos. No había sensado de la onda $\mathrm{P}$, por lo que se aumentó la sensibilidad del electrodo auricular y el dispositivo funcionó adecuadamente. Al día siguiente la paciente tenía mayor disnea, ingurgitación yugular y edemas en miembros inferiores. Se auscultó por primera vez un fuerte roce pericárdico sobre la región precordial. En el ecocardiograma se vio un

Trabajo aceptado: 24 de noviembre de 2004 
derrame pericárdico de $5 \mathrm{~mm}$ sin colapso ventricular derecho. El cable del electrodo estaba dentro del tracto de salida del ventrículo derecho. La proteína C-reactiva era de $153 \mathrm{mg} / \mathrm{dl}$, el fibrinógeno de $659 \mathrm{mg} / \mathrm{dl}$ y la VSG de $57 \mathrm{~mm}$ en la primera hora. Los anticuerpos antinucleares, anticuerpos antimúsculo cardiaco, complemento y factor reumatoide fueron negativos. Los anticuerpos frente Coxsackie B1 a B6 y Echovirus fueron también negativos. La paciente fue tratada con diuréticos y prednisona $(30 \mathrm{mg} /$ día). Su estado mejoró rápidamente. Dos semanas después, el roce pericárdico y el derrame pleural habían desaparecido y los marcadores inflamatorios se habían normalizado. La dosis de prednisona fue reduciéndose gradualmente a los dos meses hasta suspender, con recurrencia de los síntomas (dolor torácico pleurítico y elevación de los marcadores de inflamación) dos meses después. Se trató con un nuevo curso de prednisona sin recurrencias posteriores.

\section{DISCUSIÓN}

Los datos clínicos más relevantes de los casos publicados de SPIV están representados en la Tabla I. En todos los pacientes se observó cardiomegalia en la radiografía de tórax y/o derrame pericárdico (leve a severo) en el ecocardiograma. La VSG en la primera hora osciló de 20 a $145 \mathrm{~mm}$ y la proteína C-reactiva de 32 a $251 \mathrm{mg} / \mathrm{dl}$. En cuatro casos se realizó una toracocentesis. Los leucocitos predominantes en el derrame pleural fueron linfocitos (55-90\%) o eosinófilos (70\%). El contenido proteico fue normal o ligeramente elevado. También en cuatro casos fue necesario realizar pericardiocentesis o pericardiotomía quirúrgica debido a inestabilidad hemodinámica, drenando 600-800 $\mathrm{ml}$ de líquido pericárdico estéril serohemorrágico. Histológicamente el pericardio mostraba fibrosis e infiltración linfocitaria focal (5). En dos casos la forma de presentación fue un taponamiento cardiaco $(5,10)$. En el tratamiento del SP se han utilizado fármacos antiinflamatorios no esteroideos o prednisona con excelentes resultados. La dosis inicial de prednisona osciló entre 20 y 40 $\mathrm{mg}$ /día. Los síntomas y reactantes inflamatorios mejoraron rápidamente. El derrame pericárdico desapareció en 1 á 8 semanas. Los síntomas recurrieron en 3 episodios no tratados $(5,13)$, uno de los cuales desarrolló una pericarditis constrictiva que precisó una extensa pericardiectomía diez meses después de la presentación inicial del SPIV (5). Los otros tres casos que recibieron sólo tratamiento sintomático evolucionaron sin incidencias $(4,10,12)$. El SPIV se inicia probablemente tras una perforación transitoria del ventrículo derecho, que produce un pequeño hemopericardio (responsable de la fiebre y el dolor torácico inicial) y varias semanas después, por un mecanismo autoinmune, la inflamación pleuropericárdica característica del síndrome (4). No obstante, ésta hipótesis no está bien confirmada, pues sólo se ha demostrado perforación del ventrículo derecho en un caso (7), y no se detectaron anticuerpos antimúsculo cardiaco en los cuatro casos en los que se determinaron. La disfunción del marcapasos no es una característica del SPIV. Sólo en el presente caso se produjo una disfunción del marcapasos en el momento del diagnóstico.

El diagnóstico de SPIV es clínico y depende de la aparición de signos y síntomas característicos poco después de la implantación del marcapasos endovenoso y probablemente pasa desapercibido muchas veces. La incidencia de pericarditis leve tras la implantación de marcapasos con electrodo auricular en una serie de 123 pacientes fue del

TABLA I

DATOS CLÍNICOS RELEVANTES DE LOS CASOS PUBLICADOS

\begin{tabular}{|c|c|c|c|c|c|c|c|c|c|c|c|c|}
\hline Edad/Sexo & Semana * & $\begin{array}{l}\text { * Macapasos } \\
\text { Temp./tipo/elec. auricular }\end{array}$ & $\begin{array}{l}\text { Temparatura } \\
\left({ }^{\circ} \mathrm{C}\right)\end{array}$ & IY & Edemas & $\begin{array}{c}\text { Pulso } \\
\text { paradójico }\end{array}$ & $\begin{array}{c}\text { Roce } \\
\text { pericárdico }\end{array}$ & $\begin{array}{l}\text { Derrame } \\
\text { pleural }\end{array}$ & Intervención & Tratamiento & Evolución & Ref. \\
\hline $72 / \mathrm{N}$ & 12 & $+/ \mathrm{WVI} /-$ & 38,3 & - & + & - & + & Derecho & - & - & RC a las 6 semanas & 8 \\
\hline $62 / \mathrm{V}$ & 4 & $-/ \mathrm{WVI} /-$ & 38.5 & + & + & + & + & - & Pericardiocentesis & $\begin{array}{l}\text { Paracetamol } \\
\text { Diuréticos }\end{array}$ & $\begin{array}{l}\text { Pericarditis } \\
\text { constrictiva }\end{array}$ & 9 \\
\hline $75 / M$ & 2 & -/DDD/ fijado & 38.4 & + & - & - & + & Bilateral & Pericardiocentesis & Indometacina & RC a las 2 semanas & 10 \\
\hline $79 / M$ & 2 & +/DDD/pasivo & 39.5 & - & - & - & & - Izquierdo & Toracocentesis & Prednisolona & $\begin{array}{l}\text { Fiebre sin tto durante } \\
37 \text { dias. RC } 2 \text { meses } \\
\text { más tarde }\end{array}$ & 12 \\
\hline $79 / M$ & 1 & -/DDD/ fijado & $38-39$ & - & - & - & - & Izquierdo & Toracocentesis & Prdensiona & RC a las 8 semanas & 13 \\
\hline $84 / M$ & 3 & -/DDD/pasivo & Febrícula & - & - & + & - & Izquierdo & $\begin{array}{l}\text { Ventana } \\
\text { pericárdica }\end{array}$ & - & RC tras la cirugía & 14 \\
\hline $69 / M$ & 1 & -/VDDM/ pasivo & 37 & & + & + & - & +lzquierdo & Toracocentesis & Prednisolona & RC a las 8 semanas & 15 \\
\hline $68 / \mathrm{V}$ & 3 & +/WVI/- & 38 & & - & - & - & +lzquierdo & - & Sintomático & RC a las 4 semanas & 16 \\
\hline $71 / \mathrm{V}$ & 1 & +/DDD/ fijado & $37-39$ & - & - & + & + & Bilateral & Pericardiotomía & $\begin{array}{l}\text { Sintomático } \\
\text { Indometacina } \\
\text { Prednisolona }\end{array}$ & $\begin{array}{l}3 \text { episodios: tras las } \\
\text { implantaciones y la } \\
\text { pericardiotomía. RC } \\
\text { con prednislona a las } \\
4 \text { semanas }\end{array}$ & S \\
\hline $60 / M$ & 1 & -/DDDR/ fijado & $38-39$ & - & - & - & - & Bilateral & Toracocentesis & Naproxeno & RC a las 8 semanas & 11 \\
\hline $78 / M$ & 2 & +/VDD/pasivo & 37.5 & + & + & - & + & Derecho & - & Prdenisona & $\begin{array}{l}\text { RC a las } \\
8 \text { semanas } \\
\text { Recurrencia a } \\
\text { los } 2 \text { meses. }\end{array}$ & $\begin{array}{l}\text { Presente } \\
\text { caso }\end{array}$ \\
\hline
\end{tabular}

Elec. auricular: electrodo auricular. IY: ingurgitación yugular. M: mujer. RC: resolución clínica. Temp: marcapasos temporal. V: varón

* Intervalo de tiempo en semanas desde la implantación del marcapasos a la aparición de los síntomas. 
$4,9 \%$ (14). Por eso, los casos publicados de SPIV -10 en 27 años-, probablemente representan sólo los de curso más complicado. El SPIV debe tenerse en cuenta en el diagnóstico diferencial de la fiebre y el dolor pleurítico tras la implantación de un marcapasos endovenoso, junto a la infección de la herida quirúrgica o del marcapasos, a la perforación ventricular o a las enfermedades virales o autoinmunes intercurrentes.

\section{Bibliografía}

1. Ito T, Engle MA, Goldberg HP. Postpericardiotomy syndrome following surgery for nonrheumatic heart disease. Circulation 1958; 17: 549556.

2. Dressler W. The post-myocardial infarction syndrome: A report of 44 cases. Arch Intern Med 1959; 103: 28-42.

3. Peters RW, Scheinman MM, Raskin S, Thomas AN. Unusual complications of epicardial pacemakers. Am J Cardiol 1980; 45: 1088-1094.

4. Kaye D, Frankl W, Arditi LI (1975) Probable postcardiotomy syndrome following implantation of a transvenous pacemaker: Report of the first case. Am Heart J 90 (5): 627-30.

5. Foster CJ. Constrictive pericarditis complicating an endocardial pacemaker. Br Heart J 1982; 47: 497-9.

6. Snow ME, Agatston AS, Kramer HC, Samet P. The postcardiotomy syndrome following transvenous pacemaker insertion. PACE 1987; 10: 934-6.

7. Lau CP, Fong PC, Tai YT, Li JPS, Chui OCY. Postpericardiotomy syndrome complicating transvenous dual-chamber rate-adaptive pacing: Diagnosis aided y transesophageal echocardiography. Am Heart J 1992; 123: $1388-90$.

8. Hargreaves M, Bashir Y Postcardiotomy syndrome following transve- nous pacemaker insertion. Eur Heart J 1994; 15: 1005-7.

9. Goutal H, Baur F, Bonnevie L, Monnier G, Le Blainvaux M, Brion R. Syndrome pleuro-pericardique; une complication rare de la stimulation cardiaque par voie endocavitaire. Archives des maladies du coeur et des vaisseaux 1995; 88 (12): 1901-3.

10. Miller GL, Coccio EB, Sharma SC. Postpericardiotomy syndrome and cardiac tamponade following transvenous pacemaker placement. Clin Cardiol 1996; 19: 255-6.

11. Sarriá Cepeda C, Suárez Fernández C, Castrillo García JM, Romero Olabarrieta J. Síndrome postpericardiectomía tras la implantación de un marcapasos endocárdico. Med Clin (Barc) 1998; 111 (2): 78-9.

12. Bajaj BPS, Evans K, Thomas P. Postpericardiotomy syndrome following temporary and permanent transvenous pacing. Postgrad Med J 1999; 75 (884): 357-8.

13. Sasaki A, Kobayashi H, Okubo T, Namatame Y, Yamashina A. Repeated postpericardiotomy syndrome following a temporary transvenous pacemaker insertion, a permanent transvenous pacemaker insertion and surgical pericardiotomy. Jpn Circ J 2001; 65: 343-4.

14. Greene TO, Portnow AS, Huang SKS. Acute pericarditis resulting from an endocardial active fixation screw-in atrial lead. PACE 1994; 17: 21-5. 